\title{
An Improved Degree Based Condition for Hamiltonian Cycles
}

\author{
Lenin Mehedy ${ }^{1}$, Md. Kamrul Hasan ${ }^{1}$ and Mohammad Kaykobad ${ }^{2}$ \\ ${ }^{1}$ Department of Computer Engineering, Kyung Hee University, South Korea. \\ ${ }^{2}$ Department of Computer Science and Engineering, North South University, Dhaka, Bangladesh. \\ Email: ${ }^{1}\left\{\right.$ lenin, kamrul\}@oslab.khu.ac.kr, ${ }^{2}$ kaykobad@northsouth.edu
}

\begin{abstract}
A Hamiltonian cycle is a closed path through all the vertices of a graph. Since discovering whether a graph has a Hamiltonian path or a Hamiltonian cycle are both NP-complete problems, researchers concentrated on formulating sufficient conditions that ensure Hamiltonicity of a graph. A recent paper [Rahman M. Sohel and Kaykobad M., "On Hamiltonian Cycles and Hamiltonian Paths", Information Processing Letters 94(2005), 37-51] presents distance based sufficient conditions for the existence of a Hamiltonian path. In this paper we establish that the same condition forces Hamiltonian cycle to be present excepting for the case where end points of a Hamiltonian path is at a distance greater than 2.
\end{abstract}

Keywords: Hamiltonian cycle; Hamiltonian path; Graphs, Combinatorial problems

\section{Introduction}

We consider only simple graphs- graphs that do not contain loops or multiple edges. Every reference in this paper to a path or a cycle implies simple path or simple cycle. A Hamiltonian cycle is a closed path passing through every vertex of a graph. A graph containing a Hamiltonian cycle is said to be simply Hamiltonian. Naturally every Hamiltonian graph contains a Hamiltonian path and not necessarily vice versa. In fact, even when a Hamiltonian path for a graph $\mathrm{G}$ is given as an instance, finding a Hamiltonian cycle is equally difficult a task. Named after Sir William Rowan Hamilton, this problem traces its origins to the 1850s [7]. It is one of the six well-known problems that constituted the class of NP-Complete problems in the initial days of the theory of computational complexity. Scientists established NP-Completeness of the problem in very special classes of graphs [6]. So theoretically it appears a very difficult task to formulate an easily computable characterization of Hamiltonicity.

Tremendous amount of research have been done to find necessary and sufficient conditions for a graph to be Hamiltonian (see [7] for survey). Hamiltonicity of graphs have been studied in the 
perspective of independent set [12], dominating circuit [8], k-ordered Hamiltonicity [10], 2-trail (a trail that uses every vertex at most twice) [5], density (size, degrees, neighborhood conditions of a graph etc.)[7], [14], toughness [2], [3], forbidden subgraphs [4], multiple Hamiltonian cycles [9], alternating Hamiltonian cycle (a properly colored Hamiltonian cycle in which adjacent edges have different colors in a graph $G^{c}$ with colored edges) [1] etc. Rahman and Kaykobad [13] considered the shortest distance between the pair of non-adjacent vertices along with their degree sum to give sufficient conditions for Hamiltonian path in a graph.

In this paper we would like to establish that, conditions given in Rahman and Kaykobad[13] does ensure the existence of Hamiltonian cycles excepting in case when end vertices of a Hamiltonian path is exactly at a distance of 2 .

The rest of the paper is organized as follows: in Section 2 we present some necessary results to establish our claim. Section 3 describes the main results. Section 4 discusses significance of our findings and in Section 5 we indicate a direction for future research.

\section{Preliminaries}

Before presenting some of the relevant conditions of Hamiltonicity existing in the literature, we need to introduce and define some of the notations that will be used throughout this paper. Given a graph $G(V, E)$ and for a vertex $u \in V$, we mean by $d(u)$ the degree of $u$ in $G$. If $P(u, v)=\left(u=u_{0}, u_{1}, \ldots, u_{k}=v\right)$ is a path joining $u$ and $v$ in $G$, then the length of path $P(u, v)$ is $k$, i.e. the number of edges in $P$. By $\delta(u, v)$, we denote the length of a shortest path between $\mathrm{u}$ and $\mathrm{v}$ in G. On the other hand, we denote a Hamiltonian path with end vertices $\mathrm{u}$ and $\mathrm{v}$ by $H(u, v)$. If the vertices of $G$ are indexed by natural numbers then a path $P(i, l)$ may be denoted by

$\langle i \rightarrow j-k \rightarrow l\rangle \mid i, j, k, l \in V$ where by - sign we indicate a jump from natural sequence of vertices and by $\rightarrow$ sign we indicate a path corresponding to increasing or decreasing sequence between indices on both sides of the sign.

Now we are ready to list some of the relevant results available in the literature for the existence of Hamiltonian cycles or paths in graphs. 
Theorem 1.1 (Ore [11]). If $d(u)+d(v) \geq n$ for every pair of distinct non-adjacent vertices $u$ and $v$ of $G$, then $G$ is Hamiltonian.

Theorem 1.2 (Rahman and Kaykobad [13]). Let $G=(V, E)$ be a connected graph with $n$ vertices and $P$ be a longest path in $G$ having length $k$ and with end vertices $u$ and $v$. Then the following statements must hold:

(a) Either $\delta(u, v)>1$ or $P$ is a Hamiltonian path contained in a Hamiltonian cycle.

(b) If $\delta(u, v) \geq 3$ then $d_{P}(u)+d_{P}(v) \leq k-\delta(u, v)+2$.

(c) If $\delta(u, v)=2$, then either $d_{P}(u)+d_{P}(v) \leq k$ or $P$ is a Hamiltonian path and there is a Hamiltonian cycle.

Theorem 1.3 (Rahman and Kaykobad [13]). Let $G=(V, E)$ be a connected graph with $n$ vertices such that for all pairs of distinct non-adjacent vertices $u, v \in V$ we have $d(u)+d(v)+\delta(u, v) \geq n+1$. Then $G$ has a Hamiltonian path.

For ensuring Hamiltonian cycles, Ore's conditions force the graph to contain at least $\left\lfloor\frac{n^{2}}{4}\right\rfloor$ edges in the graph. But with the inclusion of the length of the shortest path in Theorem 1.3, there is a possibility of sparser graphs qualifying for containing Hamiltonian paths. In [13] it has been further asserted that:

Lemma 1.1 Let $G$ be a simple graph with $n$ vertices and $u, v$ be distinct non-adjacent vertices of $G$ with $d(u)+d(v) \geq n$. Then $\delta(u, v)=2$.

This Lemma 1.1 along with Lemma 3.2 in [13] also implies the validity of Ore's theorem. In this note we extend the results of [13] further to include existence of a Hamiltonian cycle in graphs with larger shortest paths.

\section{Main Results}

First of all we note that for the existence of Hamiltonian cycles, graph G must be free of cutvertices and cut-edges. By $\widetilde{G}$ we denote graphs without cut edges and cut vertices that also satisfy the 
hypothesis of Theorem 1.3. Now we reformulate Theorem 1.3 in the following way to assert that graph $\widetilde{G}$ is indeed Hamiltonian.

Theorem 1.4 Existence of a Hamiltonian path $H(u, v)$ in $\widetilde{G}$ with $\delta(u, v) \geq 3$ implies that $\widetilde{G}$ is Hamiltonian.

Existence of a Hamiltonian path in a graph $\widetilde{G}$ is ensured by Theorem 1.3. To prove Theorem 1.4, we have the following Lemma 1.2.

Lemma 1.2: For $H(u, v)$ in $\widetilde{G}, \delta(u, v) \leq 3$.

Proof: Let us assume for clarity of arguments that $\mathrm{u}$ is denoted by 1 and $\mathrm{v}$ is denoted by $\mathrm{n}$ and all vertices along Hamiltonian path $H(u, v)$ in $\widetilde{G}$ are denoted by $2,3 \ldots \mathrm{n}-1$ and it will be used throughout this paper. We also use $u$ and $l$ interchangeably as $v$ and $n$. We prove Lemma 1.2 by contradiction. Let us assume that $\delta(u, v) \geq 4$. Then we have the following two cases depending on the existence of cross edges like $(u, r),(v, s) \in E$ and $r>s$.

Case 1: Let us consider a graph with no cross edges like $(u, r),(v, s) \in E$ with $r>s$. Then there are at least $(\delta(u, v)-3)$ vertices among the $(n-2)$ vertices in the graph to which neither $\mathrm{u}$ nor $\mathrm{v}$ is connected (Fig. 1). Then we have

$$
d(u)+d(v) \leq n-2-(\delta(u, v)-3)
$$

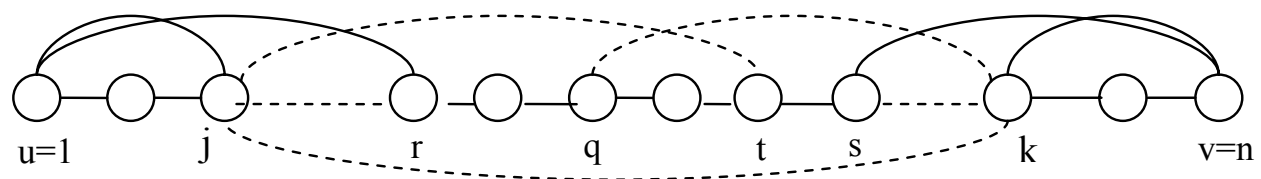

Fig. 1. A possible graph $\widetilde{G}$ with $\delta(u, v)=7$

Now, according to the hypothesis of Theorem 1.3 we have,

$$
\begin{aligned}
& d(u)+d(v)+\delta(u, v) \geq n+1 \quad \forall(u, v) \notin E \\
& \Rightarrow d(u)+d(v) \geq n+1-\delta(u, v)=(n-2)-(\delta(u, v)-3)) \\
& \Rightarrow d(u)+d(v) \geq(n-2)-(\delta(u, v)-3))
\end{aligned}
$$

Then from Eq (3.1) and Eq. (3.2) we conclude that, 
$d(u)+d(v)=n-2-(\delta(u, v)-3)$

and hence all the vertices excepting $(\delta(u, v)-3)$ are connected to either $\mathrm{u}$ or $\mathrm{v}$.

Now, it should be noted that we may avoid cut vertices and cut edges in such a graph only by adding one edge like $(j, k) \mid 1<j<r$ and $s<k<n$ (see Fig 1), which makes $\delta(u, v)=3$ through the path $\langle u \rightarrow j \rightarrow k \rightarrow n\rangle$. Otherwise, we may have edges $(j, t),(k, q) \mid 1<j<r$ and $r<q<t<s$ and $s<k<n$, which makes $\delta(u, v)$ shorter by $\max [(|t-r|-1),(|s-q|-1)]$. Since $\min [\max [(|t-r|-1),(|s-q|-1)]]=1$ and thus contradicting our assumption of $\delta(u, v) \geq 4$.

Case 2: Now we consider a graph $\widetilde{G}$ with cross edges $(u, r),(v, s) \in E$ with $r>s$. Without loss of generality, we assume that $d(u)=2$ and $(u, j),(u, r) \in E$ (Fig. 2), where $\mathrm{r}$ is the nearest possible vertex of $v$ to which $u$ can be connected (Fig. 2). This is the only way by which $u$ and $v$ can have maximum degrees in total because otherwise for each pair of cross edges $(u, r),(v, s) \in E \mid r>s$, we will have $(\delta(u, v)-3)$ number of vertices non-adjacent to both $\mathrm{u}$ and $\mathrm{v}$.

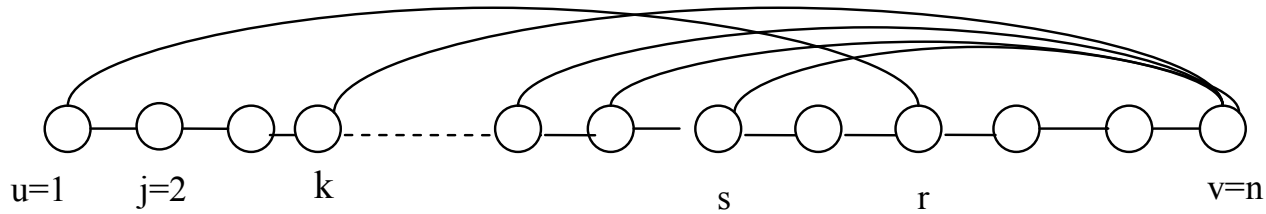

Fig. 2. A possible simple graph $\widetilde{G}$ with $\delta(u, v)=4$

Here (see Fig. 2), $|k-j|=|r-s|=|n-r|=\delta(u, v)-3$, Then we get $d(u)+d(v)=n-2-3(\delta(u, v)-3)$, which implies $d(u)+d(v)+\delta(u, v)=n-7-2 \delta(u, v)$. But $n-7-2 \delta(u, v)<n+1$ and it is a contradiction to our hypothesis. Hence we have proved that the graph $\widetilde{G}$ cannot have diameter greater than 3.

Proof of Theorem 1.4: According to Lemma 1.2, it suffices to prove the statement of Theorem 1.4 for the case when $\delta(u, v)=3$. Now $\delta(u, v)=3$ implies that no vertex $w \in V$ can be adjacent to $u, v$ at the same time since then $\langle u \rightarrow w \rightarrow v\rangle$ would have been a path of length 2. 


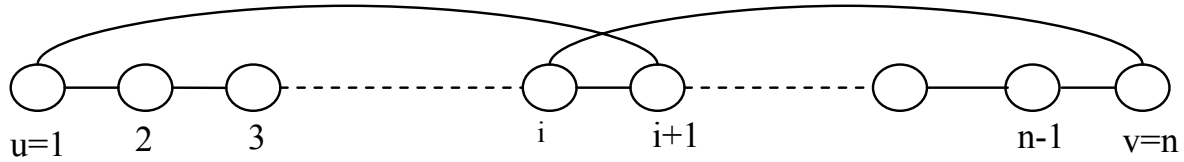

Fig. 3. Existence of crossover edge $(1, i+1)$ and $(i, n)$

Existence of cross over edges $(1, i+1),(i, n) \in E$ as depicted in Fig. 3 above implies existence of a Hamiltonian cycle, in particular $\langle 1-(i+1) \rightarrow n \overparen{i} \rightarrow 1\rangle$ is a Hamiltonian cycle. So we assume that there is no crossover edge. Then it is similar to case 1 in the proof of Lemma 1.2 and hence from Eq. (3.3) we have $d(u)+d(v)=n-2-(\delta(u, v)-3)$, which implies $d(u)+d(v)=n-2$ for $\delta(u, v)=3$. Hence each of the vertices is connected either to $\mathrm{u}$ or tos $\mathrm{v}$.

Let $\mathrm{k}$ be the highest index such that $(1, k) \in E$, then $(k-1, n)$ is a cross over edge, and hence $(k-1, n) \notin E$. Since each vertex must be connected either to 1 or $\mathrm{n}$ exclusively, it then means $(1, k-1) \in E$. The same argument leads to the statement that $(1, i) \in E, \forall i \leq k$. According to our assumption $(1, k+1) \notin E$, so the vertex $k+1$ must be connected to n. Again similar argument will result in $(j, n) \in E, \forall j \geq k+1$. Now the corresponding graph looks like Fig.4.

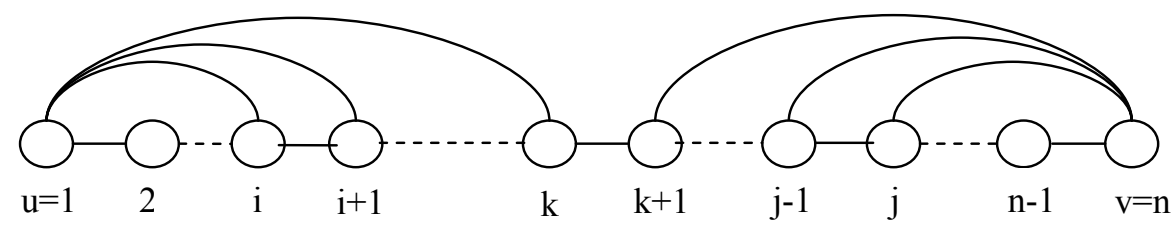

Fig. 4. A graph $\widetilde{G}$ without crossover edges with $\delta(u, v)=3$

Since $\widetilde{G}$ is free of cut-vertices and cut-edges, then at least one of the following conditions must hold:

(i) $(i, j) \in E, \quad 1<i<k<k+1<j<n$, in which case $\langle 1 \rightarrow i \widetilde{T} j \rightarrow \widetilde{T}(j-1) \rightarrow(i+1)-1\rangle$ is a Hamiltonian cycle (Fig.4), or

(ii) $(k, j),(k+1, i) \in E, 1<i<k<k+1<j<n$, in which case the desired Hamiltonian cycle is $\langle 1 \rightarrow i \widetilde{T}(k+1) \rightarrow(j-1) \widetilde{T} n \rightarrow j \widetilde{T} k \rightarrow(i+1) \widetilde{T}\rangle$ (Fig 4). So this proves our theorem.

Remarks: It may be interesting to observe that with Hamiltonian path $H(u, v)$ and $\delta(u, v)=2$, a certain pattern of graph $\widetilde{G}$ does not ensure a Hamiltonian cycle. In this pattern, vertex 1 and $n$ are 
connected to every alternate vertex in $\{2,3, \ldots, \mathrm{n}-1\}$ and all vertices that are non-adjacent to both 1 and $n$ are connected to all vertices that are adjacent to both 1 and $n$ (Figs 5 and 6).

In such a graph with even number of vertices, we have one pair of vertices which are adjacent to each other and also connected to any of the end vertices 1(or n) (vertex 2 and 3 in Fig. 6). It should be noted that we will consider such graphs where there is no cross over edges like $(1,3)$ and $(2, n)$ because otherwise the graph is Hamiltonian due to the cycle $\langle\sqrt{3} 3 \rightarrow n-2 \rightarrow 1\rangle$ (Fig. 6).

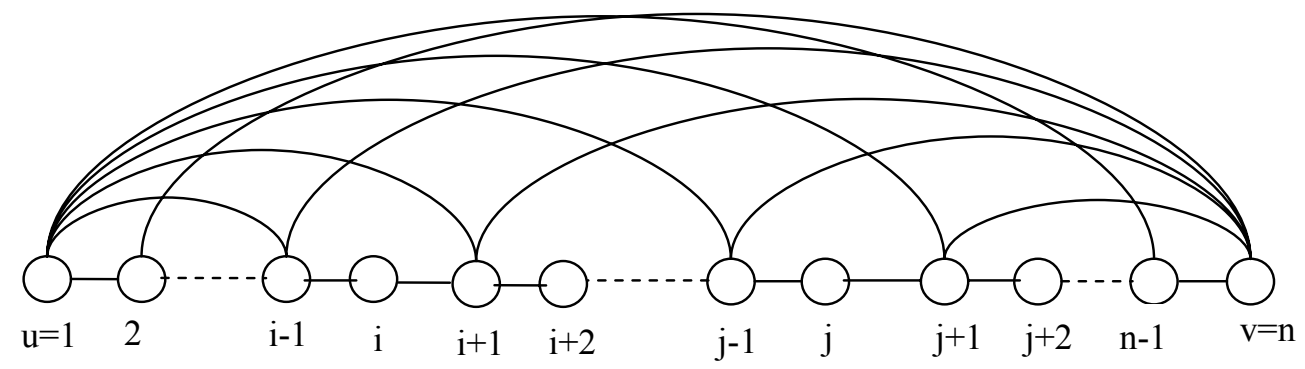

Fig. 5. A graph $\widetilde{G}$ with $\delta(u, v)=2$ and odd number of vertices

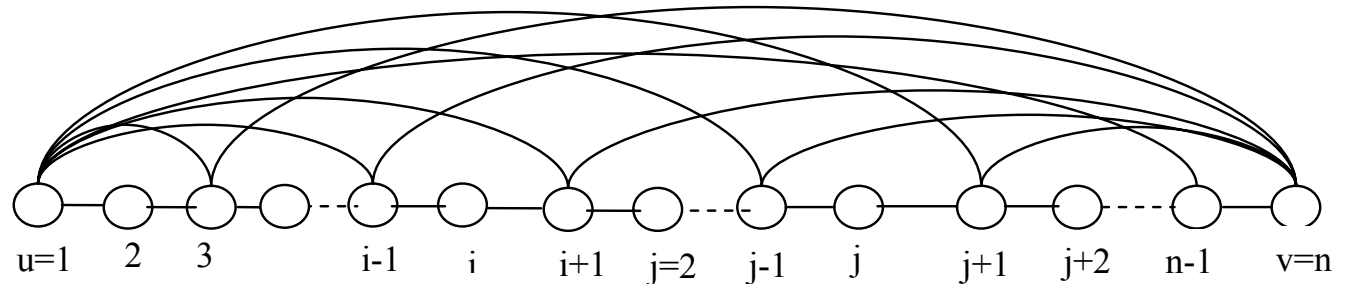

Fig. 6. A graph $\widetilde{G}$ with $\delta(u, v)=2$ and even number of vertices

Now we show that increasing the degree of any of the end vertices 1(or n) in Figures 5 and 6 will ensure Hamiltonicity. The only possible way to increase the degree of vertex $l$ (or $n$ ) is to connect vertex 1 (or $n$ ) with any vertex $i$, which is also non adjacent to $n$ in this pattern of graph. Then $\langle\widetilde{1} i \rightarrow n \widetilde{(}(i-1) \rightarrow 1\rangle$ is our desired Hamiltonian cycle.

Also we may note that, if we connect vertex $i$ and $j$ where $i$ and $j$ are not adjacent to $l$ and $n$, we will again have a Hamiltonian cycle $\langle 1 \rightarrow i \widetilde{R} j \rightarrow n \widetilde{R}(j-1) \rightarrow(i+1) \widetilde{R} 1\rangle$.

\section{Significance of our results}

The conditions derived in this paper appears superior to that of Ore [11] since it ensures Hamiltonicity 
in a graph demanding lesser number of edges. We show that our conditions require at least $\left\lfloor\frac{n}{4}\right\rfloor$ fewer edges than Ore's requirement of $\left\lfloor\frac{n^{2}}{4}\right\rfloor$ edges to ensure Hamiltonicity of a graph. So we have the following theorem.

Theorem 1.5 : A graph satisfying Theorem 1.4 will require at least $\left\lfloor\frac{n}{4}\right\rfloor$ fewer edges than Ore's requirement of $\left\lfloor\frac{n^{2}}{4}\right\rfloor$ edges to ensure Hamiltonicity.

Proof: From the hypothesis of Theorem 1.3 we have,

$$
\begin{aligned}
& d_{i}+d_{j}+\delta(u, v) \geq n+1, \quad \forall(i, j) \notin E \\
& \Rightarrow \sum_{i=1}^{n}\left(n-1-d_{i}\right) d_{i}+\sum_{(i, j \notin E)} \delta(i, j) \geq(n+1)\left(\left(\begin{array}{l}
n \\
2
\end{array}\right)-E\right) \\
& \Rightarrow 2 E(n-1)-\sum_{i=1}^{n} d_{i}^{2}+\sum_{(i, j \notin E)} \delta(i, j) \geq(n+1)\left(\left(\begin{array}{l}
n \\
2
\end{array}\right)-E\right)
\end{aligned}
$$

Since we know that $\sum_{i=1}^{n} d_{i}=2 E, \sum_{i=1}^{n} d_{i}^{2}$ will be minimum when $d_{i}=\frac{2 E}{n}, \forall i=1,2, \ldots n$.

Hence we have $\sum_{i=1}^{n} d_{i}^{2} \geq \frac{4 E^{2}}{n}$. Again $\sum_{(i, j \notin E)} \delta(i, j) \geq 2\left(\left(\begin{array}{l}n \\ 2\end{array}\right)-E\right)$ because the minimum

distance between any pair of non-adjacent vertices is 2 . Then we get,

$$
\begin{aligned}
& 2 E(n-1)-\frac{4 E^{2}}{n}+2\left(\left(\begin{array}{l}
n \\
2
\end{array}\right)-E\right) \geq(n+1)\left(\left(\begin{array}{l}
n \\
2
\end{array}\right)-E\right) \\
& \Rightarrow 8 E^{2}-E\left(6 n^{2}-6 n\right)+n^{2}(n-1)^{2} \leq 0 \\
& \Rightarrow\left(\frac{n^{2}}{4}-\frac{n}{4}\right) \leq E \leq\left(\frac{n^{2}}{2}-\frac{n}{2}\right) \\
& \Rightarrow E \geq\left(\frac{n^{2}}{4}-\frac{n}{4}\right)
\end{aligned}
$$

Hence our conditions require $\left\lfloor\frac{n}{4}\right\rfloor$ fewer edges than Ore's requirement of $\left\lfloor\frac{n^{2}}{4}\right\rfloor$ edges to ensure 
Hamiltonicity.

\section{Conclusion}

In this paper we have presented a degree based sufficient condition for Hamiltonicity in a graph. It is also established that inclusion of the concept of shortest paths demands lesser number of edges than that required by Ore's conditions to ensure Hamiltonicity of a graph. It would be interesting to investigate the possibility of applying all pairs shortest path matrix to formulate better conditions.

\section{References}

[1] Barr O., "Properly Coloured Hamiltonian Paths In Edge-Colored Complete Graphs Without Monochromatic Triangles", Ars Combinatoria 50(1998), 316-318.

[2] Bauer D., Broersma H.J., Schmeichel E., "More Progress On Tough Graphs - The Y2K Report", Electronic Notes in Discrete Math. 11 (July 2002).

[3] Böhme T., Harant, J., Tkáč, M., "More Than 1-Tough Chordal Planar Graphs Are Hamiltonian", J. Graph Theory 32(1999), 405-410.

[4] Duffus D., Gould R. J., Jacobson M.S., "Forbidden Subgraphs And The Hamiltonian Theme", The Theory and Applications of Graphs, ed. by Chartrand, Alavi, Goldsmith, Lesniak and Lick, (1981), 297-316.

[5] Ellingham M.N., Zha X., Zhang Y., "Spanning 2-Trails From Degree Sum Conditions”, J. Graph Theory, Vol. 45, Iss. 4 (2004), 298-319.

[6] Garey M.R. , Johnson D.S., "Computers And Intractability: A Guide To The Theory Of NPCompleteness", W.H. Freeman and Company, New York, 1979.

[7] Gould R. J., "Advances On The Hamiltonian Problem - A Survey", Graphs and Combinatorics, Vol. 19, Num. 1, March 2003, 7 - 52.

[8] Gould R.J., Hynds E., “A Note On Cycles In 2-Factors Of Line Graphs”, Bull. of the I.C.A., Vol. 26(1999), 46-48.

[9] Horak P., Stacho L., "A Lower Bound On The Number Of Hamiltonian Cycles”, Discrete Math. 222(2000), no. 1-3, 275-280.

[10] Kierstead H., Sárközy G., Selkow S., “On K-Ordered Hamiltonian Graphs”, J. Graph Theory, 32 (1999), 17-25.

[11] Ore O., "Note On Hamiltonian Circuits", Amer. Math. Monthly 67(1960), 55.

[12] Plotnikov A.D., "One Criterion Of Existence Of A Hamiltonian Cycle", Reliable Comput. 4 (1998), 199-202.

[13] Rahman M. Sohel , Kaykobad M., "On Hamiltonian Cycles And Hamiltonian Paths", Information processing Letters 94(2005), 37-51

[14] Rahman M. Sohel, Kaykobad M., Rahman M. Saifur, "A New Sufficient Condition for the Existence of Hamiltonian Paths", $20^{\text {th }}$ International Conference on Computers and their Applications (CATA), 2005. 